\title{
Optimizing adaptable systems for future uncertainty
}

\author{
Daniel Straub \& Olga Špačková \\ Engineering Risk Analysis Group, Technische Universität München, Germany \\ straub@tum.de, olga.spackova@tum.de
}

\begin{abstract}
Demands on structures and infrastructures change over their service life and cannot be predicted with certainty. Adaptable (or flexible) infrastructure designs are thus potentially beneficial, enabling easier adjustments of the systems at a later stage. However, systematic quantitative investigations and corresponding recommendations are missing. In (Špačková and Straub 2016), we present a framework for such an analysis, which is based on sequential decision processes. In this contribution, we summarize the approach and focus on the interpretation of flexibility. We show that the framework enables quantification of the value of flexibility, to answer the question: what is the maximum amount that should be spent additionally to ensure system flexibility? Two case studies illustrate that this value is strongly dependent on a number of factors, in particular on the types of uncertainty present and the amount of future information collected in the future.
\end{abstract}

Keywords: planning, infrastructure, risk, POMDP, decision making, sustainability, adaptability

\section{Introduction}

Most structures and infrastructure are built to last, with projected service life times of 50 years or more. However, these systems are subject to changing demands from environment and users over their service life. Bridges are deteriorating and are subject to possibly increasing traffic loads, demands on dwater infrastructure are affected by changing climates and population development and industrial facilities undergo changes in user requirements (Hall et al. 2014; Yzer et al. 2014). If these systems cannot be adapted to the new demands, they may become inefficient or obsolete. On the other hand, increasing the flexibility or adaptability of engineering systems is typically associated with additional costs, and it may turn out to be unnecessary in the long run if demands are not changing. To further complicate the matter, safety margins against future changes in demand may be built into systems as an alternative to building adaptable systems. These margins also come at a cost though, and it is necessary to find a trade-off among safety, adaptability and risk.

Such an optimization problem can be formalized by sequential decision analysis, which was first developed by economists and was later enhanced in the field of artificial intelligence (Raiffa and Schlaifer 1961; Kochenderfer et al. 2015). In (Špačková and Straub 2016), we 
show that the theory and the available mathematical tools are ideally suited to model infrastructure systems under uncertain future demands. The approach can account for the fact that adaptable systems may be adjusted when demands are changing or when new information is available in the future. In contrast to alternative approaches, most of which are based on realoptions analysis (e.g. Neufville et al 2006), sequential decision analysis allows to consistently address all uncertainty and decision alternatives in the process, and also can account for partial observability of the relevant processes. To facilitate the modeling process and communication, the decision process is represented by an influence diagram, similar to the proposal of (Nishijima 2016).

A special focus of this contribution is on investigating the effect of a system's flexibility. It has been pointed out in the literature that flexible system designs can be advantageous under future uncertainties, such as climate change uncertainty or demand uncertainty (Hallegate 2009). Intuitively, this appears reasonable, as flexible systems can be adapted in the future with limited cost. However, formal quantitative investigations of the effect of flexibility in the context of infrastructure planning are missing. To enable such analysis, we propose a measure of flexibility in (Špačková and Straub 2016). Through sequential decision analysis, one can then derive a value of flexibility and make recommendations on optimal strategies for dealing with future uncertainty. In particular, the relation between a system's flexibility and the initial safety margin can be derived. As we show, this relation depends on a number of factors, not least the amount of information that can be obtained in the future.

The generic concepts are illustrated by application to infrastructure subject to demand uncertainty and to flood management systems under climate change uncertainty.

\section{Adaptable or flexible engineering systems}

Adaptable or flexible systems are designed such that they are easily adjusted to changing demands (Ross et al. 2008; Saleh et al. 2009). Examples include pipes with additional capacity for future transmission cables, buildings with structural systems that enable flexible floor plans or flood defense systems where land for future extension is reserved. Because such flexibility comes at an additional cost, an optimization should be carried out to understand if it pays off. Furthermore, if a system is more flexible, the optimal design of the system might change (if transmission cables can be added later, fewer cables might be installed initially).

We propose to measure flexibility through the cost of establishing capacity. Conceptually, Fig. 1 shows two systems with higher and lower flexibility.

\subsection{A measure of flexibility}

To formalize the analysis of flexibility, we proposed a quantitative measure $\varphi$ of flexibility in (Špačková and Straub 2016 and Špačková et al. 2015). The measure is based on the costs of establishing and upgrading a system. Let $c(v)$ denote the cost of establishing a system capacity $v$ initially. In a flexible system, the cost $\Delta c$ of increasing the system capacity from a value $v^{\prime}$ to a higher $v^{\prime \prime}$ should be comparable to the difference of the costs for establishing $v^{\prime \prime}$ and $v^{\prime}$ initially. Therefore, one can write this upgrading cost $\Delta c$ as 


$$
\Delta c\left(v^{\prime}, v^{\prime \prime}\right)=c\left(v^{\prime \prime}\right)-\varphi \cdot c\left(v^{\prime}\right)
$$

It follows that the measure of flexibility is defined as

$$
\varphi=\frac{c\left(v^{\prime \prime}\right)-\Delta c\left(v^{\prime}, v^{\prime \prime}\right)}{c\left(v^{\prime}\right)} .
$$

All costs in Eqs. (1) and (2) are undiscounted values, since the system flexibility measure should not depend on time. The actual net present value of upgrading the system from $v^{\prime}$ to $v^{\prime \prime}$ might therefore be lower than $\Delta c$ according to Eq. (1).

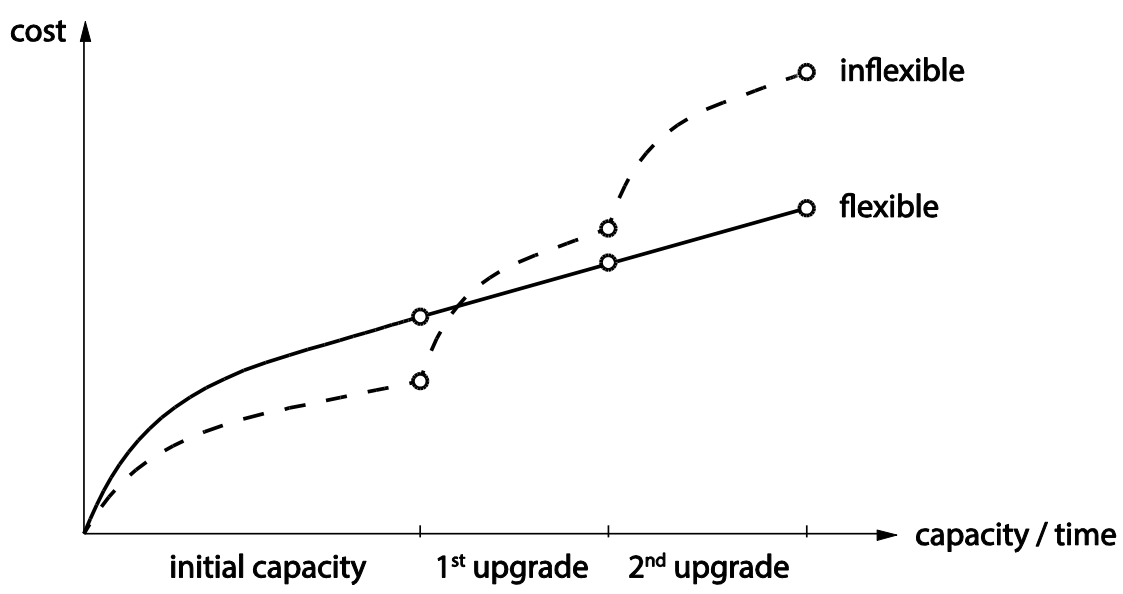

Fig. 1: Illustration of the total development cost for a flexible vs an inflexible system.

Shown are the costs associated with establishing the initial capacity and the costs associated with the $1^{\text {st }}$ and $2^{\text {nd }}$ upgrade conditional on existing levels of capacity. While the inflexible system is typically cheaper initially, it may lead to larger lifetime costs when updates become necessary.

\section{Sequential decision analysis}

The optimization of infrastructure system capacity over time can be approached with sequential (Bayesian) decision analysis, which originated in mathematical economics (Raiffa and Schlaifer 1961) and was further developed in artificial intelligence and planning (e.g. Kaelbling et al. 1998, Kochendoerfer 2015). Decisions are optimized following the expected utility principle, which here corresponds to a minimization of expected present value life cycle costs. Uncertainties are modelled probabilistically, and the effect of future information on the uncertainties is accounted for by Bayesian analysis.

Following (Špačková and Straub 2016), infrastructure capacity planning can be generically represented by a partially observable Markov decision process (POMDP). This only requires the demand process to be modelled as a Markov process ${ }^{1}$. The generic POMDP, and its special case, the MDP (Markov decision process), are represented by the influence diagrams

\footnotetext{
${ }^{1}$ As discussed in (Špačková and Straub 2016), this is not a strong limitation, since most non-Markovian processes can be transformed into a Markovian process by augmenting the state space.
} 
(IDs) in Fig. 2. An ID is an extension of Bayesian networks that includes decision and utility (cost) nodes; the former are represented by squares, the latter by diamond-shaped nodes. IDs can mostly be understood intuitively, the detailed semantics are described e.g. in (Jensen and Nielsen 2007). An important aspect of an ID are the links pointing towards a decision node. They reflect the flow of information, as they indicate that the parent node is known at the time of making the decision.

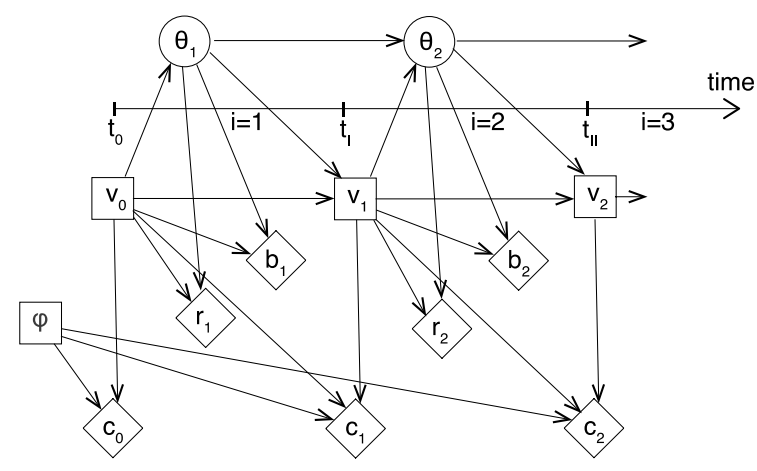

(a)

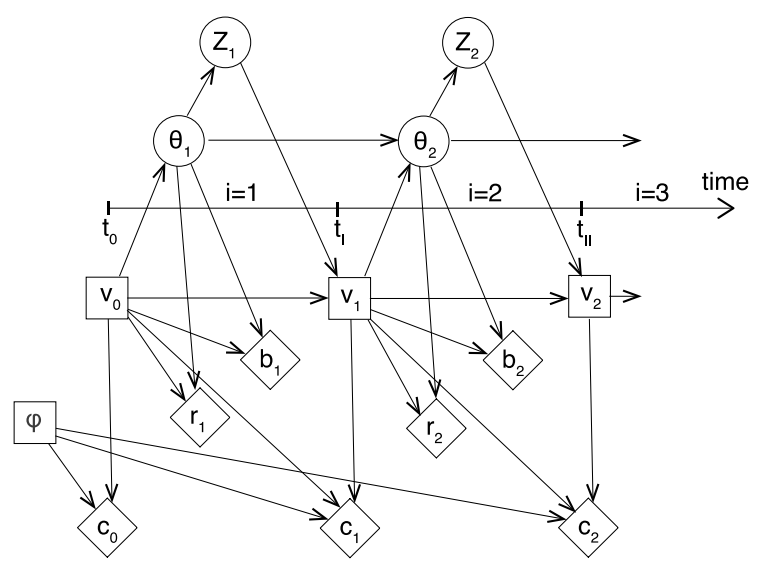

(b)

Fig. 2: Influence diagram representing the general infrastructure capacity planning problem. (a) Markov decision process (MDP). (b) Partially observable Markov decision process (POMDP). The variables at each time step $t$ are: $\theta_{t}$ : demand, $Z_{t}$ : measurement, $v_{t}$ : capacity, $\varphi$ : flexibility, $b_{t}$ : benefits, $r_{t}$ : risk (associated with demands exceeding capacity), $c_{t}$ : cost of system update. (Figure from Špačková and Straub 2016).

The ID of Fig. $2 \mathrm{~b}$ shows the POMDP model, in which the demand node $\theta_{t}$ cannot be observed directly before a decision is made at $t+1$. Instead, an indicator variable $Z_{t}$ is observed, which represents partial information on the demand variable. Unfortunately, this partial observability leads to computationally challenges in identifying optimal decision policies. An introduction to POMDP is found in (Kochenderfer et al. 2015). POMDP has previously been applied to planning of inspections in deteriorating structure and infrastructure (e.g. Madanat 1993; Corotis et al. 2005; Papakonstantinou and Shinozuka 2014; Memarzadeh and Pozzi 2016). A special case of this model arises when the demand at any time $t$ can be observed with certainty. In this case, the POMDP reduces to the MDP (Fig. 2a), which is substantially easier to solve. For details on the computation of such POMDP or MDP models in the context of planning in adaptable infrastructure systems, the reader is referred to (Špačková and Straub 2016).

In a POMDP/MDP, the decision to be taken at each time is described by a policy, which describes the action to be taken conditional on the available information. In an MDP, this information is the current state of demand $\varphi_{t}$, in the POMDP, this is the current state of belief, which summarizes all past observations. If these policies are not changing with time, they are called stationary policies. An approximate solution to a POMDP can be found by defining a stationary policy through a limited number of parameters $\mathbf{d}$ (a heuristic), computing the expected total utility by means of a Monte Carlo approach for a given heuristic, and then performing an optimization over $\mathbf{d}$. Such approaches are common in risk-based inspection planning (e.g. Straub and Faber 2006). 


\section{$4 \quad$ Numerical illustrations}

\subsection{Case 1: Infrastructure capacity}

In this example, we consider a generic model for infrastructure capacity planning, where the demand at present is observable with high accuracy. Examples of such problems include transportation infrastructure, water resource systems or electrical power networks. The problem setting is summarized by the ID of Fig. 3. In Fig. 3a, the actual model is shown where the demand at each time step is defined conditional on the trend $\tau$, which reflects the mean change in the demand. An equivalent POMDP is obtained by replacing the common variable $\tau$ with identical copies $\tau_{t}$ (corresponding to an augmentation of the state space).

(a) Original decision model

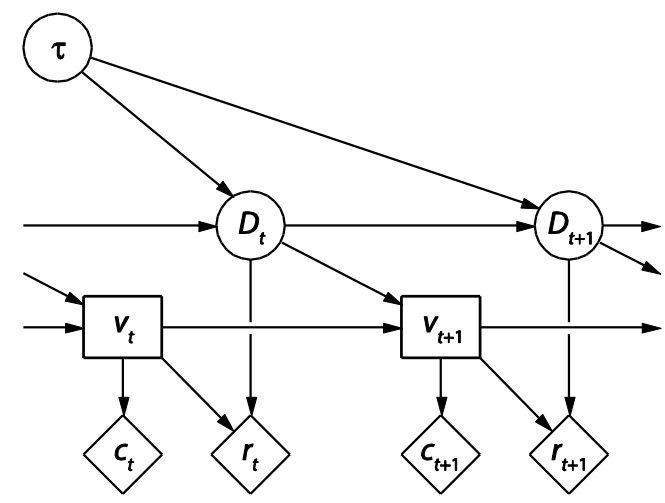

(b) Equivalent POMDP

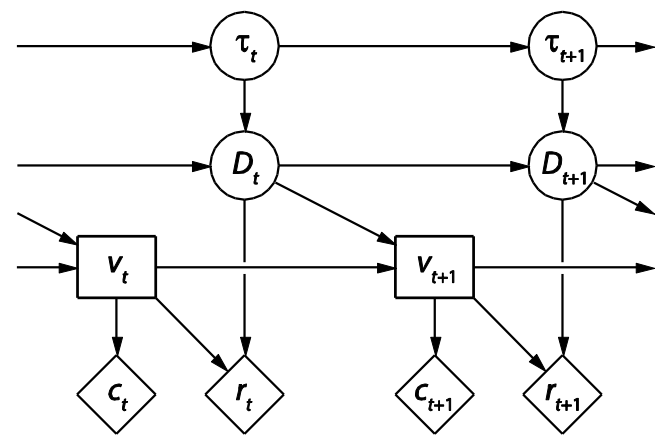

Fig. 3: Influence diagram representing the investigated infrastructure capacity planning problem. $\tau$ is the trend in the demand, $D_{t}$ is the system demand, $v_{t}$ is the system capacity, $c_{t}$ is the cost associated with upgrading the system, and $r_{t}$ is the cost associated with the demand exceeding the capacity.

The remaining uncertainty in observations of the demand $D_{t}$ can be neglected, therefore there is a link from $D_{t}$ to the decision node $v_{t+1}$, indicating direct observability of $D_{t}$. Note that the process is nevertheless only partially observable, because the trend variables $\tau_{t}$ can be inferred only indirectly.

The considered service life is 50 years, with a $2 \%$ discounting rate. For the numerical investigation, the parameters of the model are according to Table 1 . The trend is modelled by a discrete random variable with three possible scenarios. The demand is modelled as a lognormal random process.

It is assumed that decisions on upgrading system capacity are made every 5 years. A reduction of capacity is not considered, as there are no benefits associated with such a reduction. 
Table 1: Parameters of the infrastructure capacity case study.

\begin{tabular}{lll}
\hline Parameter & Type & Description \\
\hline Trend $\tau$ & Discrete random variable & $p_{\tau}(0)=1 / 3, p_{\tau}(0.01)=1 / 3, p_{\tau}(0.02)=1 / 3$ \\
Demand $D_{t}$ & Lognormal random process & $D_{0}=1$ \\
& & $\ln D_{t} \mid \ln D_{t-1} \sim N\left(\ln D_{t}+\tau, \sigma_{\Delta D}\right)$ \\
& & $\sigma_{\Delta D}=0.05$ \\
Capacity $v_{t}$ & Decision process & optimization parameter \\
Capacity cost & Function & $c(v)=\ln (1+v)$ \\
Cost of demand & Function & $r_{t}=D_{t}-v_{t}$, if $D_{t}>v_{t}$, else 0 \\
Discount rate $\rho$ & Deterministic & 0.02 \\
\hline
\end{tabular}

The optimal life-cycle strategies are identified by means of the heuristic approach in combination with Monte Carlo sampling.

The stationary decision policy for $t>0$ is parametrized as follows:

- Extend capacity when the demand times a tolerance parameter $\alpha$ exceeds capacity, i.e. if $\alpha \cdot D_{t}>v_{t}$.

- If the capacity is extended, then to a value $\gamma \cdot D_{t}$. Here, $\gamma$ represents the overdesign of a system modification.

The initial capacity is selected as $v_{0}=S F \cdot D_{0}$, where $S F$ is a safety factor (initial overdesign). Therefore, the optimization parameters are $\mathbf{d}=[S F ; \alpha, \gamma]$.

In Fig. 4, the expected net present value of the life-cycle costs in function of flexibility and the initial safety factor are shown. Costs decrease with increasing flexibility, as expected. The optimum safety factor, i.e. the initial overdesign, increases as the flexibility decreases, from a value of around 1.4 (for $\varphi=1$ ) to 3 (for $\varphi=0$ ).

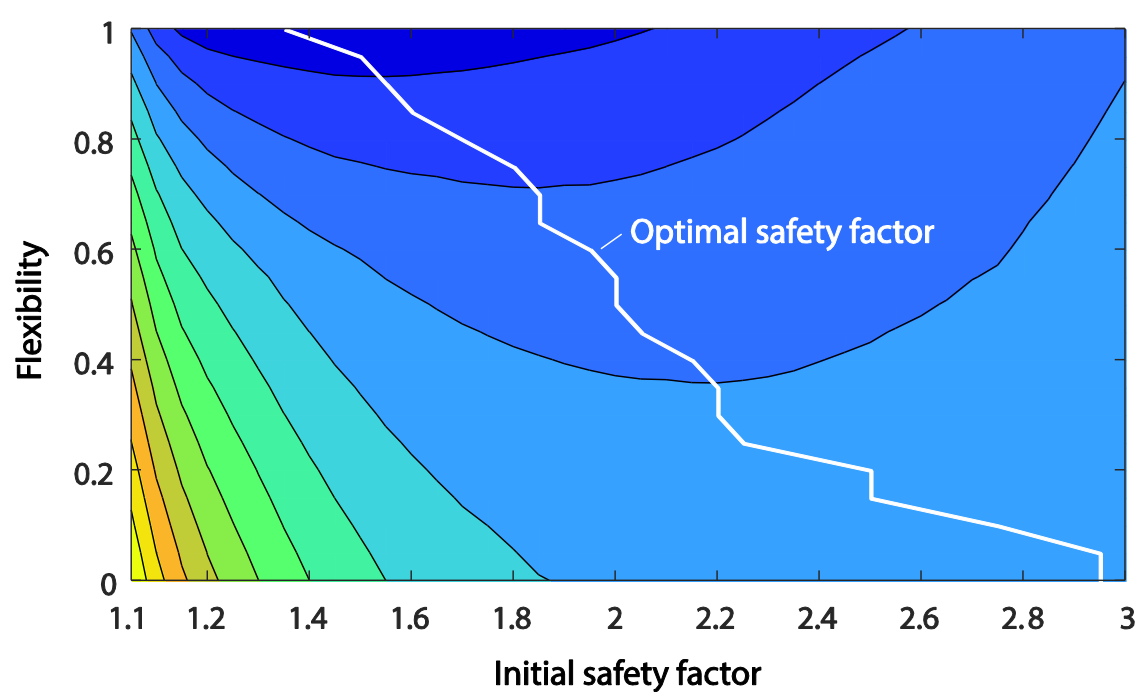

Fig. 4: Expected net present value of life-cycle costs in function of the initial safety factor and the flexibility of the system, together with the optimal safety factor.

In Fig. 5, the optimal expected net present life-cycle costs are plotted for varying system flexibilities (these are the values found along the white line of Fig. 4). The largest costs are 
incurred for the inflexible system. The reduction in costs for higher values of flexibility reflects the value of flexibility.

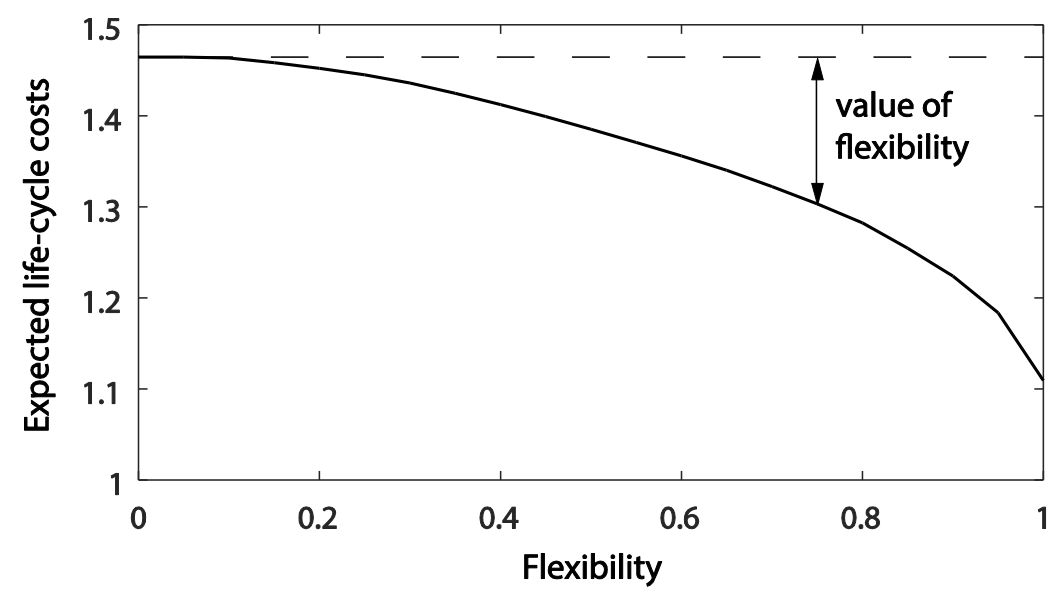

Fig. 5: Optimal expected net present value of the life-cycle cost in function of flexibility. The difference relative to the value achieved with flexibility zero is the value of flexibility.

The temporal distribution of optimal expected costs varies in function of the system flexibility (Fig. 6). In case of the inflexible system, the optimal strategy is to invest initially, and then accept the possibility of costs because of insufficient capacity towards the end of service life. For the fully flexible system, the costs are most equally distributed over the service life. Whenever the capacity is insufficient, or if it is likely that the capacity will become insufficient in the next years, the system is upgraded.
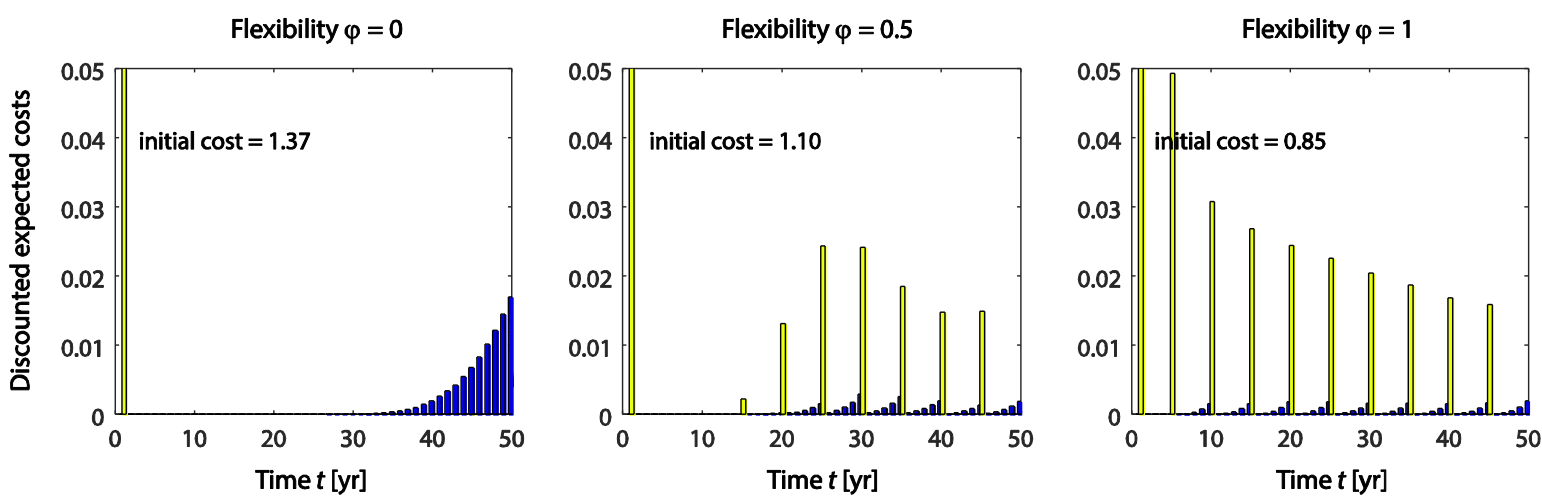

Fig. 6: Distribution of expected discounted costs over the lifetime for the different flexibilities, when the optimal management strategy is implemented. The yellow (lighter) bars correspond to cost associated with building or upgrading the system $c_{t}$, and the blue (darker) bars are the expect costs associated with the demand $D_{t}$ exceeding the capacity $v_{t}$. 


\subsection{Case 2: Disaster risk management}

Disaster risk mitigation infrastructures, such as flood defences, are designed to protect society from extreme events. The frequency of extreme events is not directly observable - many years of observations are in fact needed to derive the frequency accurately (Dittes et al. 2016). This problem is intensified when the frequency and characteristic of extreme events changes in time (is non-stationary), e.g. due to climate change.

The following example on planning of flood mitigation measures under climate change uncertainty is taken from (Špačková and Straub 2016). The presentation here differs the one in the original paper. Three climate scenarios are considered: A - no change in extreme discharge frequency, $\mathrm{B}$ - moderate increase of frequency of extreme discharges and $\mathrm{C}$ - significant increase. These climate scenarios correspond to trend values $\tau=0,1,2$, respectively. In the future, observed annual maximum discharges will be applied to update the probabilistic believes on the climate scenarios.

The model corresponds to the model shown in Fig. 3. Decisions on flood protection capacity are revised every 30 years, the total planning horizon is 90 years. Definitions of the utilized variables are provided in Table 2. The risk and cost functions are defined in the original paper.

Table 2: Parameters of the flood protection case study.

\begin{tabular}{lll}
\hline Parameter & Type & Description \\
\hline Climate trend $\tau$ & Discrete RV & $p_{\tau}(0)=1 / 3, p_{\tau}(1)=1 / 3, p_{\tau}(2)=1 / 3$ \\
Ann.max discharge $D_{t}$ & Continuous RV & $D_{t} \mid \tau \sim \operatorname{Gumbel}(1200+2 \cdot \tau \cdot t, 960+1.6 \tau)$ \\
Capacity $v_{t}$ & Decision process & optimization parameter \\
Discount rate $\rho$ & Deterministic & 0.02 \\
\hline
\end{tabular}

In Table 3, the optimal initial designs of the flood protection system are summarized for stationary conditions (neglecting the effect of climate change, i.e., assuming that the probability of scenario A is 1) and under consideration of the uncertain effects of climate change. The latter results are shown for varying flexibility $\varphi$. The difference between flexible and inflexible systems is very low, indicating that the flexibility has limited value in this case.

Table 3: Optimal initial design of the flood protection system excluding and including uncertain climate impact for different flexibilities.

\begin{tabular}{lcccc}
\hline & Neglecting climate & \multicolumn{3}{c}{ Including climate change uncertainty } \\
& change & $\varphi=0$ & $\varphi=0.5$ & $\varphi=1$ \\
\hline Design discharge & $4800 \mathrm{~m}^{3} / \mathrm{s}$ & $5240 \mathrm{~m}^{3} / \mathrm{s}$ & $5240 \mathrm{~m}^{3} / \mathrm{s}$ & $5220 \mathrm{~m}^{3} / \mathrm{s}$ \\
Design return period & $220 \mathrm{yr}$ & $400 \mathrm{yr}$ & $400 \mathrm{yr}$ & $380 \mathrm{yr}$ \\
\hline
\end{tabular}



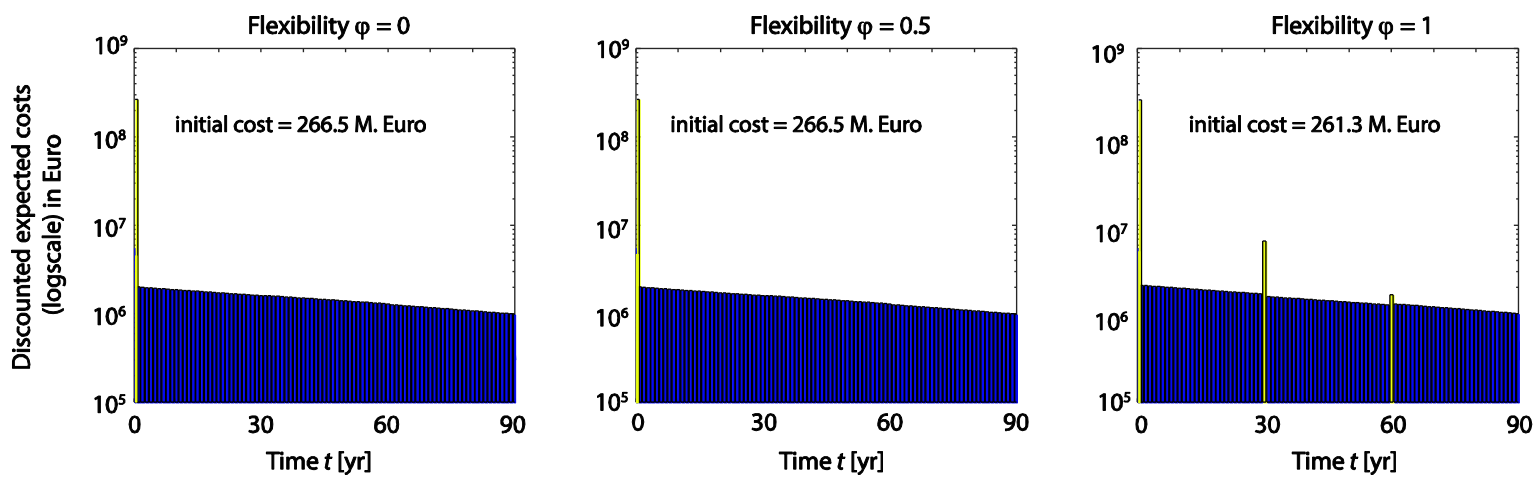

Fig. 7: Distribution of expected discounted costs over the lifetime for the different flexibilities, when the optimal management strategy is implemented. The yellow (lighter) bars correspond to cost associated with building or upgrading the flood defense, and the blue (darker) bars are the discounted flood risks.

\section{$5 \quad$ Concluding remarks}

We present a framework that enables the investigation of the effect of adaptability (flexibility) in infrastructure systems in a systematic and quantitative manner. Adaptability is frequently mentioned as a potentially effective strategy to deal with uncertain climate change and other future changes and uncertainties. However, numerical investigations into its effect are lacking, which is the aim of this research. To enable a generalization of results from individual case studies, we propose a measure of flexibility. Taking basis in sequential decision analysis, it is then possible to quantify the value of flexibility.

The results of the two case studies, and others reported in (Dittes et al. 2016) and (Špačková and Straub 2016), indicate that the value of flexibility can be fundamentally different depending on a number of factors, which include the amount of uncertainty and the possibility for future learning (reducing uncertainty), the mean predicted changes of the system, the discounting rate as well as the cost and risk functions. Comparing the two presented examples, one can observe that the flexibility has a significant value in the infrastructure capacity example, where the learning process is strong. This is in contrast to the second example, where the uncertainty is on extremes, which are generally hard to predict. The presented example does underestimate the true capability for learning, because it is not accounted for improvements in climate models and other information that can be used to improve flood predictions. Nevertheless, the value of information will be limited also under modified assumptions, because increasing flood protection capacity is a no-regret strategy. I.e., a conservative design has benefits under any future change, which is not the case in the first example.

\section{References}

Corotis, R.B., Hugh Ellis, J., Jiang, M., 2005. Modeling of risk-based inspection, maintenance and life-cycle cost with partially observable Markov decision processes. Struct. Infrastruct. Eng. 1, 75-84. 
Dittes B., Špačková O., Straub D. (2016) Managing uncertainty in flood predictions: Flexible protection strategies vs safety factors. Working paper, ERA Group, TU München, Germany.

Hall, J.W., Otto, A., Tran, M., Barr, S., Alderson, D., 2014. A National Model for Strategic Planning of Infrastructure Systems. American Society of Civil Engineers: 2821-2829.

Hallegatte, S., 2009. Strategies to adapt to an uncertain climate change. Glob. Environ. Change, Traditional Peoples and Climate Change 19, 240-247.

Jensen, F. V. and T. D. Nielsen (2007). Bayesian networks and decision graphs, Springer.

Kaelbling, L.P., Littman, M.L., Cassandra, A.R., 1998. Planning and acting in partially observable stochastic domains. Artif. Intell. 101, 99-134.

Kochenderfer, M.J., Amato, C., Chowdhary, G., How, J.P., Reynolds, H.J.D., Thornton, J.R., Torres-Carrasquillo, P.A., Üre, N.K., Vian, J., 2015. Decision Making Under Uncertainty: Theory and Application. MIT Press.

Madanat, S. (1993). Optimal infrastructure management decisions under uncertainty. Transportation Research Part C: Emerging Technologies 1(1): 77-88.

Memarzadeh, M., Pozzi, M., 2016. Integrated Inspection Scheduling and Maintenance Planning for Infrastructure Systems. Comput.-Aided Civ. Infrastruct. Eng. 31: 403-415.

Neufville, R. de, Scholtes, S., Wang, T., 2006. Real Options by Spreadsheet: Parking Garage Case Example. J. Infrastruct. Syst. 12: 107-111.

Nishijima, K., 2015. Concept of decision graphical framework for optimising adaptation of civil infrastructure to a changing climate. Struct. Infrastruct. Eng. 12(4): 477-483.

Papakonstantinou, K. G., Shinozuka M. 2014. Optimum inspection and maintenance policies for corroded structures using partially observable Markov decision processes and stochastic, physically based models. Probabilistic Engineering Mechanics 37: 93-108.

Raiffa, H. and R. Schlaifer (1961). Applied statistical decision theory. Division of Research, Graduate School of Business Administration, Harvard University.

Ross, A.M., Rhodes, D.H., Hastings, D.E., 2008. Defining changeability: Reconciling flexibility, adaptability, scalability, modifiability, and robustness for maintaining system lifecycle value. Syst. Eng. 11, 246-262. doi:10.1002/sys.20098

Saleh, J.H., Mark, G., Jordan, N.C., 2009. Flexibility: a multi-disciplinary literature review and a research agenda for designing flexible engineering systems. J. Eng. Des. 20, 307-323.

Špačková O., Dittes B., Straub D. (2015). Risk-based optimization of adaptable protection measures against natural hazards. Proc. ICASP 12, Vancouver, Canada.

Špačková O., Straub D. (2016). Bayesian models for long-term adaptation decisions. Working paper, ERA Group, TU München, Germany.

Straub D., Faber M.H. (2006). Computational Aspects of Risk Based Inspection Planning. Computer-Aided Civil and Infrastructure Engineering, 21(3), pp. 179-192.

Yzer, J.R., Walker, W.E., Marchau, V., Kwakkel, J.H., 2014. Dynamic adaptive policies: A way to improve the cost-benefit performance of megaprojects. Environ. Plan. B Plan. Des. 41. 